Marcia Bicudo de Paula

Almério de Castro Gomes
Departamento de Epidemiologia. Faculdade de Saúde Pública. Universidade de São Paulo. São Paulo, SP, Brasil

Correspondência | Correspondence:

Marcia Bicudo de Paula

Departamento de Epidemiologia

Faculdade de Saúde Pública, Universidade de São Paulo

Av. Doutor Arnaldo, 715

01246-904 São Paulo, SP, Brasil

E-mail: bicudo@usp.br

\section{Culicidae (Diptera) em área sob influência de construção de represa no Estado de São Paulo}

\section{Culicidae (Diptera) in a dam construction area in the state of São Paulo, Brazil}

\section{RESUMO}

OBJETIVO: Descrever a fauna Culicidae em área sob impacto da modificação ambiental causada pela construção de represa.

MÉTODOS: Foi estudada a área onde estava sendo construída a Represa Porto Primavera no município de Presidente Epitácio, SP. As capturas dos culicídeos foram realizadas mensalmente, em seis pontos exploratórios antes do seu enchimento e em um ponto fixo, um ano antes (1997-1998) e outro depois do primeiro enchimento (1999-2000). Mosquitos adultos foram capturados com aspirador, no período matutino, e com armadilhas de Shannon e CDC, no período crepuscular vespertino. Os imaturos foram coletados por meio de conchadas nos criadouros maiores e com pipeta, nos menores.

RESULTADOS: No período anterior ao enchimento da represa foram coletados 944 exemplares de culicídeos imaturos, distribuídos em dez gêneros. O total de adultos foi de 14.932, dos quais 7.031 mosquitos (em dez gêneros) foram capturados nos pontos exploratórios e 7.901 espécimes, distribuídos em oito gêneros, no ponto fixo. Após a primeira etapa de enchimento da represa, o total de imaturos foi de 1.201 exemplares, distribuídos em quatro gêneros, e o total de mosquitos capturados foi de 5.912, distribuídos em nove gêneros. Houve redução populacional de espécies dos gêneros Aedes e Psorophora e aumento no número de espécimes dos gêneros Aedeomyia, Anopheles, Culex (Melanoconion), Mansonia e Uranotaenia.

CONCLUSÕES: As mudanças ambientais decorrentes da construção da Represa Porto Primavera favoreceram o aumento da densidade de algumas populações de culicídeos vetores e que geram incômodo, com riscos potenciais de transmissão de patógenos para humanos.

DESCRITORES: Culicidae. Represas. Impacto ambiental.

\section{ABSTRACT}

OBJECTIVE: To describe the culicidae fauna in an area impacted by environmental changes caused by the construction of a dam.

METHODS: The studied area was the Porto Primavera dam construction site in Southeastern Brazil. Monthly culicidae samples were taken in six exploratory sites in the pre-filling period and in one fi xed site, one year before (1997-1998) 
and after the first filling (1999-2000). Adult mosquitoes were captured in the morning with an aspirator and with Shannon and CDC traps during the evening twilight period. Immature stages were captured using a ladle in the larger breeding sites and with a pipette in the smaller ones.

RESULTS: In the pre-filling period of the dam, a total of 944 immature culicidae specimens, from ten species, were captured. A total of 14,932 adults were captured, including 7,031 mosquitoes from ten species in the exploratory points and 7,901 specimens from eight genera in the fixed site. After the first stage of the dam filling, the immature stages total was 1,201, from four genera, and the captured mosquitoes total was 5,912, from nine genera. There was a population reduction of the genera Aedes and Psorophora and an increase of the following species populations: Aedeomyia, Anopheles, Culex (Melanoconion), Mansonia and Uranotaenia.

CONCLUSIONS: The environmental changes caused due to the construction of the Porto Primavera Dam contributed to an increase in the population density of some culicidae vectors, causing a nuisance and the potential risk of the transmission of pathogens to humans.

\section{KEYWORDS: Culicidae. Dams. Environmental impact.}

\section{INTRODUÇÃO}

O Brasil possui rede hidrográfica vasta e densa, com alto potencial dos cursos de água para produção de energia gerada a partir da construção de grandes barragens. Os empreendimentos hidrelétricos causam impactos sociais e ambientais relacionados à remoção de populações ribeirinhas, à alteração da vazão do rio barrado, à flora e à fauna. ${ }^{7}$ Desde o século XIX, há registros do favorecimento de culicídeos por alterações ambientais quando da formação de hidrelétricas. ${ }^{9}$

As modificações ambientais podem alterar a dinâmica populacional dos culicídeos, colocando a população humana local ou migrante sob risco de contrair doenças veiculadas por esses mosquitos. Os mosquitos de comportamento antropofílico constituem possível condição de transmissibilidade de patógenos e exposição ao incômodo provocado pela atividade de picar, quando em grande abundância. ${ }^{12}$

Na década de 1980, teve início o represamento de parte do Rio Paraná para a construção da Usina Hidrelétrica de Porto Primavera (Engenheiro Sérgio Motta). A construção abrangeu os municípios de Bataiporã e Rosana, respectivamente, no Estado de Mato Grosso do Sul e São Paulo, com a inundação do complexo de lagoas da antiga Reserva Ecológica Lagoa São Paulo e suas imediações. Na região oeste do Estado de São Paulo, a construção da usina afetou os municípios de Presidente Epitácio e Caiuá, ao longo da margem esquerda do Rio Paraná e do Rio do Peixe. Presidente Epitácio representa porta de acesso ao Estado de São Paulo nas rotas de transporte rodoviário que ligam as Regiões Sul e Sudeste do País com os estados amazônicos. Dessa forma, o intenso movimento populacional entre essas regiões aumenta a vulnerabilidade das áreas que há algum tempo não apresentam transmissão de malária. Presidente Epitácio e outros municípios próximos ao Rio Paraná foram apontados como importantes focos dessa doença no Estado de São Paulo em 1980 e $1982 .{ }^{16}$

O objetivo da presente pesquisa foi descrever a influência das alterações ambientais relacionadas à Represa da Usina Hidrelétrica Porto Primavera sobre a composição da fauna Culicidae, desde o desmatamento até a primeira etapa de enchimento, visando a contribuir com uma análise ecológica de vetores biológicos de importância à saúde pública.

\section{MÉTODOS}

A área de estudo localiza-se no município de Presidente Epitácio ( $21^{\circ} 45^{\prime} 34^{\prime \prime} \mathrm{S}$ e $\left.52^{\circ} 06^{\prime} 12^{\prime \prime} \mathrm{W}\right)$ na margem esquerda do Rio Paraná. É uma extensa área inundada pela Usina Hidrelétrica de Porto Primavera, atingindo também parte da margem esquerda do Rio do Peixe. A cobertura vegetal caracteriza-se por fragmento de Floresta Estacional Semidecidual (Figura 1).*

Os locais de coleta (Figura 2) compreenderam um ponto fixo e seis pontos exploratórios. O ponto fixo situava-se no Sítio JB, localizado em frente à Reserva Lagoa São 


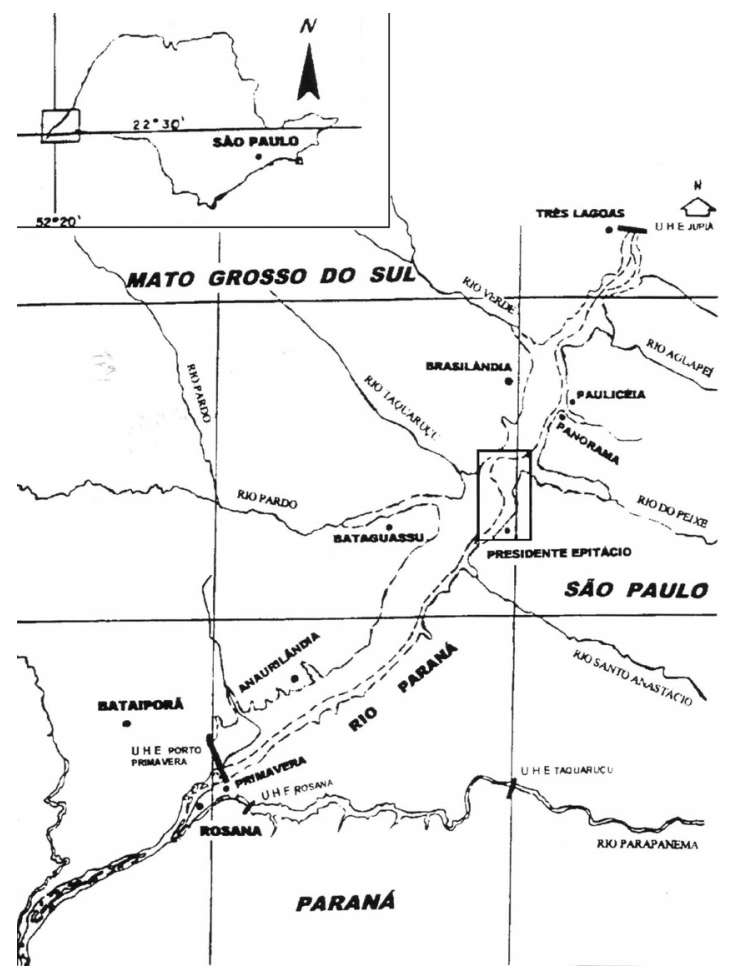

Figura 1. Localização da Usina Hidrelétrica de Porto Primavera e da área de estudo (dentro do retângulo menor) na região oeste do Estado de São Paulo.

Fonte: Companhia Energética de São Paulo, 1993.

Paulo e brejo, onde a vegetação principal é o capim colonião (Panicum maximum) e o aguapé (Eicchornia crassipes). O leito original do Rio Paraná correspondia, em 1997, à cota de $247 \mathrm{~m}$ em relação ao nível do mar. Posteriormente, entre dezembro de 1998 e janeiro de 1999, essa área foi transformada no leito do lago Porto Primavera (cota de $253 \mathrm{~m}$ ), com a inundação parcial de uma mata residual, área cultivada e domicílios.

As capturas mensais dos mosquitos adultos e imaturos no ponto fixo ocorreram nos dois períodos: julho/1997 a junho/1998 e abril/1999 a março/2000. Nos pontos exploratórios, ocorreram entre o período de julho/1997 a junho/1998, com captura dos mosquitos adultos por aspiração em julho e dezembro/1997 e janeiro e abril/1998; e com armadilha de Shannon em julho, novembro e dezembro/1997 e janeiro, fevereiro, abril e maio/1998. As técnicas de captura no ponto fixo e nos pontos exploratórios foram: aspiração, ${ }^{10}$ realizada por um coletor no período diurno e padronizada em 15 min de duração para cada coleta, em abrigos naturais e artificiais, envolvendo ecótopos de mata, campos abertos, peridomicílio e intradomicílio, antes do enchimento do lago (cota de $247 \mathrm{~m}$ ) e na margem e mata da represa após a formação do lago (cota de $253 \mathrm{~m}$ ). Capturas em armadilha de Shannon ${ }^{15}$ foram realizadas por dois coletores na margem da mata, no período vespertino, com horário da captura estratificado

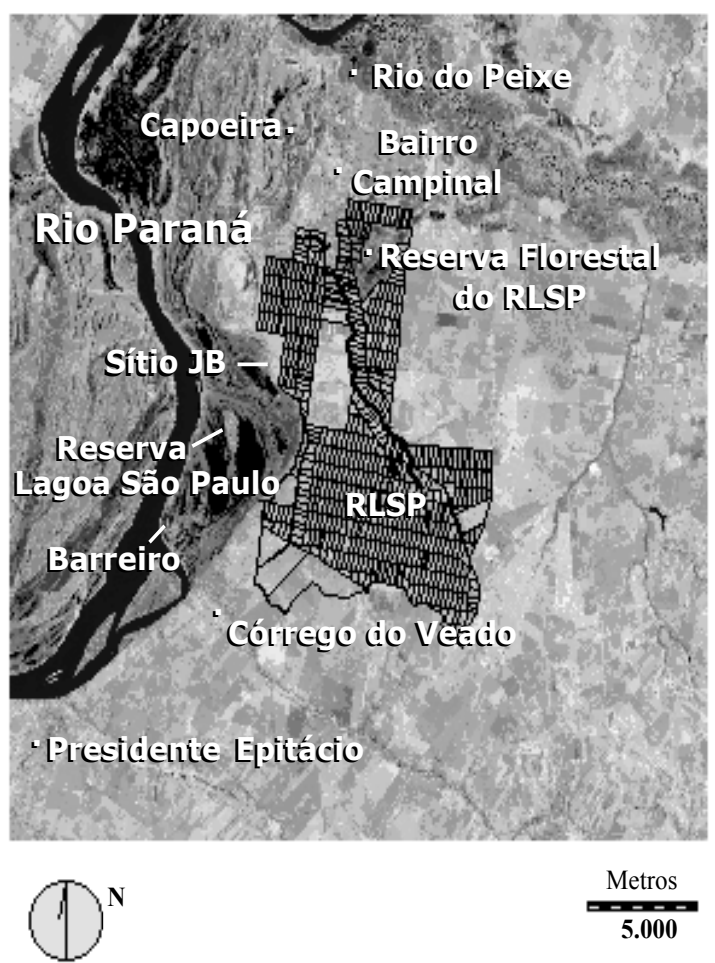

Figura 2. Localização dos pontos de coleta: fixo e exploratórios. Represa de Porto Primavera, 1997.

RLSP: Reassentamento da Lagoa São Paulo

como primeiro e segundo pré-crepúsculos, crepúsculo, primeiro, segundo, terceiro e quarto pós-crepúsculos. No ponto fixo, no período anterior ao enchimento do lago, foram instaladas seis armadilhas $\mathrm{CDC}^{6}$ por coleta em ambiente de mata (solo e copa), ambiente aberto (campo e brejo) e domicílio (intra e peri), no horário das 17:00 às 21:00 h, a cerca de um metro do solo, com exceção da copa, a $15 \mathrm{~m}$ de altura.

Nas coletas de imaturos em grandes criadouros foram padronizadas 30 conchadas de $500 \mathrm{ml}$ positivas (presença de larvas e pupas por conchada) e nos pequenos criadouros utilizaram-se pipetas. ${ }^{14}$ No período anterior ao enchimento do lago foram pesquisados sete locais, entre criadouros naturais e artificiais. Após a formação do lago, foram analisados dois criadouros naturais.

Os exemplares dos culicídeos coletados foram encaminhados para identificação ao laboratório da Faculdade de Saúde Pública da Universidade de São Paulo.

Ochlerotatus foi considerado como subgênero $\mathrm{e}^{4}$ as abreviações dos nomes genéricos dos culicídeos seguiram Reinert ${ }^{13}$ (1975).

\section{RESULTADOS}

No período anterior ao enchimento (cota $247 \mathrm{~m}$ ), 14.932 culicídeos adultos foram coletados por meio das téc- 
nicas da aspiração (5,1\%), armadilha CDC $(6,8 \%)$ e armadilha de Shannon $(88,1 \%)$ (Tabela 1$)$.

Nos pontos exploratórios foram capturados 7.031 mosquitos (Tabela 1) representados por dez gêneros (Aedeomyia, Aedes, Anopheles, Coquillettidia, Culex, Mansonia, Psorophora, Sabethes, Uranotaenia e Wyeomyia). Com a técnica de aspiração, foram coletados 138 (2,0\%) espécimes (20 machos e 118 fêmeas), registrando-se a presença de Ae. albopictus dentre uma riqueza de 20 espécies ou grupos. Por meio da armadilha de Shannon, 62 espécies ou grupos foram capturados, totalizando $6.893(98,0 \%)$ exemplares (593 machos e 6.300 fêmeas). As seguintes espécies foram capturadas exclusivamente por esta armadilha nos pontos exploratórios: An. braziliensis, An darlingi, An. evansae, An. oswaldoi, Ae.fulvus, Ae. hortator, Cx. (Melanoconion) aureonotatus, Cx. (Mel.) idottus, Cx. (Mel.) vaxus, Ps. albipes, Ps. cingulata, Sa. glaucodaemon, Wy. melanocephala e Wy. roucoyana/chalcocephala.

No ponto fixo foram capturados 7.901 mosquitos (Tabela 1) representados por oito gêneros (Aedeomyia, Aedes, Anopheles, Coquillettidia, Culex, Mansonia, Psorophora, e Uranotaenia) (Tabela 2). Na armadilha CDC foram capturados 1.011 (12,8\%) espécimes (68 machos e 943 fêmeas) de 43 espécies ou grupos. $C x$. (Culex) nigripalpus e Ur. nataliae foram espécies coletadas apenas por essa técnica. A aspiração resultou na captura de 626 (7,9\%) exemplares (215 machos e 411 fêmeas) de 51 espécies ou grupos. Com a armadilha de Shannon, capturaram-se 6.264 (79,3\%) exemplares (573 machos e 5.691 fêmeas). Considerando-se apenas o total de fêmeas capturadas nessa armadilha, a riqueza

Tabela 1. Distribuição da fauna Culicidae nos períodos anterior (cota $247 \mathrm{~m}$ ) e posterior ao enchimento (cota $253 \mathrm{~m}$ ) da Represa Porto Primavera, segundo técnicas de coleta. Represa Porto Primavera, SP, 2000.

\begin{tabular}{|c|c|c|c|c|c|c|c|c|}
\hline \multirow{3}{*}{ Técnica de coleta } & \multicolumn{6}{|c|}{ Cota $247 \mathrm{~m}$} & \multirow{2}{*}{\multicolumn{2}{|c|}{$\frac{\text { Cota } 253 \mathrm{~m}}{\text { Ponto fixo }}$}} \\
\hline & \multicolumn{2}{|c|}{ Pontos exploratórios } & \multicolumn{2}{|c|}{ Ponto fixo } & \multicolumn{2}{|c|}{ Total } & & \\
\hline & $\mathrm{N}$ & $\%$ & $\mathrm{~N}$ & $\%$ & $\mathrm{~N}$ & $\%$ & $\mathrm{~N}$ & $\%$ \\
\hline Armadilha CDC & - & - & 1.011 & 12,8 & 1.011 & 6,8 & - & - \\
\hline Aspiração & 138 & 2,0 & 626 & 7,9 & 764 & 5,1 & 425 & 7,2 \\
\hline Armadilha de Shannon & 6.893 & 98,0 & 6.264 & 79,3 & 13.157 & 88,1 & 5.487 & 92,8 \\
\hline Total & 7.031 & 100,0 & 7.901 & 100,0 & 14.932 & 100,0 & 5.912 & 100,0 \\
\hline
\end{tabular}

Tabela 2. Distribuição de adultos e imaturos da fauna Culicidae por gênero e períodos de estudo, no ponto fixo. Represa Porto Primavera, SP, 2000

\begin{tabular}{|c|c|c|c|c|}
\hline \multirow{2}{*}{$\begin{array}{l}\text { Forma } \\
\text { Gênero/Subgênero }\end{array}$} & \multicolumn{2}{|c|}{ Adultos } & \multicolumn{2}{|c|}{ Imaturos } \\
\hline & $\begin{array}{c}\text { Período anterior à } \\
\text { formação da Represa } \\
\text { Porto Primavera (\%) }\end{array}$ & $\begin{array}{c}\text { Período posterior ao } \\
\text { primeiro enchimento } \\
\text { da Represa Porto } \\
\text { Primavera }(\%)\end{array}$ & $\begin{array}{c}\text { Período anterior à } \\
\text { formação da Represa } \\
\text { Porto Primavera (\%) }\end{array}$ & $\begin{array}{c}\text { Período posterior ao } \\
\text { primeiro enchimento } \\
\text { da Represa Porto } \\
\text { Primavera (\%) }\end{array}$ \\
\hline Aedeomyia & 47,5 & 19,6 & 2,8 & $39,0^{*}$ \\
\hline Aedes & 54,2 & 1,6 & 0,6 & - \\
\hline Anopheles & 24,2 & $26,6^{*}$ & 3,7 & $16,4^{*}$ \\
\hline Coquillettidia & 43,9 & 1,9 & - & - \\
\hline Culex & 86,6 & 77,8 & 81,8 & 34,2 \\
\hline Cx. (Aedinus) & 4,4 & 0,1 & - & - \\
\hline Cx. (Culex) & 29,7 & 13,7 & 79,3 & 17,6 \\
\hline Cx. (Melanoconion) & 52,5 & $64,0^{*}$ & 2,4 & $16,6^{*}$ \\
\hline Cx. (Phenacomyia) & - & - & 0,1 & - \\
\hline Limatus & - & 0,1 & 4,6 & - \\
\hline Mansonia & 28,8 & $55,5^{*}$ & 0,2 & - \\
\hline Orthopodomyia & - & - & 0,5 & - \\
\hline Psorophora & 8,5 & 0,3 & 5,3 & - \\
\hline Sabethes & - & - & 0,2 & - \\
\hline Uranotaenia & 6,3 & $16,5^{*}$ & 0,2 & $10,4^{*}$ \\
\hline
\end{tabular}

* Culicídeos que tiveram aumento populacional (\%) no período posterior ao enchimento (cota 253m) da Represa. 
foi de 37 espécies ou grupos. A identificação dos machos foi relacionada à parte, e incluiu as espécies $C x$. (Cux.) maxi, Ma. indubitans e Ps. confinnis.

No período posterior ao primeiro enchimento (cota $253 \mathrm{~m}$ ) foram coletados 5.912 culicídeos adultos no ponto fixo, por meio das técnicas da aspiração e armadilha de Shannon (Tabela 1). Esses culicídeos representaram nove gêneros (Aedeomyia, Aedes, Anopheles, Coquillettidia, Culex, Limatus, Mansonia, Psorophora e Uranotaenia) (Tabela 2). A riqueza foi de 31 espécies ou grupos tanto para a aspiração como para a armadilha de Shannon, sendo consideradas somente as fêmeas para esta armadilha. Capturas por meio de aspiração resultaram em 425 (7,2\%) espécimes (190 machos e 235 fêmeas). Por meio dessa técnica, registrou-se a presença de Ae. aegypti e nenhum espécime de Coquillettidia foi capturado. Em armadilha de Shannon foram capturados 5.487 (92,8\%) exemplares (445 machos e 5.042 fêmeas) e registrada a presença de $C x$.(Cux.) coronator e Li. flavisetosus.

Foram coletados 944 exemplares de imaturos, de 34 espécies ou grupos e dez gêneros (Tabela 2) no período anterior ao enchimento do lago. Foi assinalada a presença de Ae. terrens, An. deaneorum, Cx. (Phenacomyia) corniger, Li. durhami e Orthopodomyia. Após a formação da Represa, foram coletados 1.201 espécimes de 27 espécies ou grupos e quatro gêneros (Tabela 2), com o encontro de An. argyritarsis e $C x$. (Mel.) clarki.

Observou-se que algumas espécies de culicídeos, entre adultos e imaturos, apresentaram aumento ou redução de sua população após o primeiro enchimento da represa, no ponto fixo (Tabela 2 ).

\section{DISCUSSÃO}

A biodiversidade da área de estudo já se encontrava alterada em função da devastação e instalação da agropecuária. Com a formação da Represa Porto Primavera, novas modificações ambientais ocorreram, interferindo nas populações de culicídeos, fato observado por outros autores..$^{11,12}$

Com a primeira elevação do nível de água (cota 253m) nas planícies dos rios Paraná e Peixe, os criadouros naturais dos gêneros Aedes e Psorophora foram alagados. Isso pode ter influenciado na baixa densidade de mosquitos adultos e ausência de imaturos dessas espécies. Entretanto, para adultos dos gêneros Anopheles, Culex (Melanoconion), Mansonia e Uranotaenia, e para os imaturos dos gêneros Aedeomyia, Anopheles, Culex (Melanoconion) e Uranotaenia observou-se elevação de sua densidade populacional, sugerindo ser resultante dos efeitos do alagamento. Possivelmente tal fato esteja relacionado às condições ecológicas favoráveis à proliferação de mosquitos desses grupos. $\mathrm{O}$ acúmulo de vegetação aquática na represa pode ter favorecido esse crescimento populacional. A expansão de macrófitas nas margens da represa ampliou a área dos culicídeos associados a essa vegetação. Neste particular, Ad. squamipennis foi uma das espécies predominantes, o mesmo sendo observado por Lopes \& Lozovei ${ }^{8}$ (1995) no norte do Estado do Paraná. Isso sugere risco potencial de circulação do vírus Gamboa (Bunyavirus) até agora transmitido primariamente por essa espécie, ${ }^{2}$ também vetora natural de malária aviária na Venezuela. ${ }^{5}$

Alguns representantes da tribo Mansoniini apresentam especificidade de associação em relação à vegetação aquática ${ }^{4}$ fato que pode explicar a redução da população de Coquillettidia e o crescimento da população de Mansonia, logo após o enchimento da represa. As espécies de Mansonia, apesar de não serem vetoras de patógenos no Brasil, causam grande incômodo à população humana $\mathrm{e}$ animal. Tadei* (1996) relatou que do total de culicídeos coletados após o enchimento do reservatório de Tucuruí (Estado do Pará), 97,1\% foram Mansonia, com predominância absoluta de Ma. titillans $(96,0 \%$ ), sendo registrado o incômodo causado por essa espécie no peridomicílio, com média de 612 mosquitos por homem/hora. Em Porto Primavera, entre os mosquitos Mansonia presentes no estudo, Ma. humeralis foi a espécie mais abundante, tendendo a representar problema semelhante ao mencionado em Tucuruí.

Anopheles darlingi encontrou condições favoráveis à sua proliferação após o enchimento da represa, fato que merece acompanhamento pois este mosquito é vetor primário de malária. ${ }^{4}$

Espécimes do grupo de $C x$. (Melanoconion) foram abundantes e podem introduzir arboviroses em ambiente antrópico. Isso porque apresentam valência ecológica suficiente para permitir evolução de hábitos no sentido de domiciliação, ${ }^{3}$ e são responsáveis pelo ciclo silvestre de transmissão enzoótica de arbovírus. ${ }^{12}$

Almirón \& Brewer ${ }^{1}$ (1996) observaram que espécies do gênero Uranotaenia foram coletadas nas margens de lagos e lagoas, associadas a espécies dos gêneros Anopheles e Culex, em vários lugares ensolarados com vegetação aquática, assim como o encontrado na Represa Porto Primavera.

A detecção de Ae. aegypti em zona rural foi um achado atípico. Ainda que possa ser considerado um encontro esporádico, essa informação sugere uma tentativa

*Tadei WP. O gênero Mansonia (Diptera: Culicidae) e a proliferação de mosquitos na usina hidrelétrica de Tucuruí. In: Magalhães SB, Brito RC, Castro ER, organizadores. Energia na Amazônia. Belém: MPEG/FPA/UNAMAZ; 1996. v. 1, p. 311-8. 
de dispersão populacional a partir de focos urbanos da cidade de Presidente Epitácio, situada a $20 \mathrm{~km}$ do local de captura.

A opção do emprego de variadas técnicas entomológicas explorando os locais aquáticos e terrestres permitiu reconhecer maior número de espécies ou grupos da fauna Culicidae. Por outro lado, as alterações ambientais decorrentes da formação do lago limitaram os locais de coleta e afetaram as populações com reflexo na alteração da

\section{REFERÊNCIAS}

1. Almirón WR, Brewer ME. Classification of immature stage habitats of Culicidae (Diptera) collected in Córdoba, Argentina. Mem Inst Oswaldo Cruz. 1996;91:1-9.

2. Dutary BE, Petersen JL, Peralta PH, Tesh RB.

Transovarial transmission of Gamboa virus in a tropical mosquito, Aedeomyia squamipennis. Am J Trop Med Hyg. 1989;40:108-13.

3. Forattini OP, Gomes AC, Kakitani I. Observações sobre mosquitos Culicidae adultos em cultivo irrigado de arroz no Vale do Ribeira, Estado de São Paulo, Brasil. Rev Saúde Pública. 1989;23:307-12.

4. Forattini OP. Culicidologia médica. São Paulo: EDUSP; 2002. v. 2.

5. Galbadon A, Ulloa G, Godoy N, Marquez E, Pulido J. Aedeomyia squamipennis (Diptera: Culicidae) vector natural de malaria aviaria en Venezuela. Bol Dir Malariol Saneam Amb. 1977;17:9-13.

6. Gomes AC, Rabello EX, Natal D. Uma nova câmara coletora para armadilha CDC-miniatura. Rev Saúde Pública. 1985;19:190-1.

7. Guimarães AE, Mello RP, Lopes CM, Alencar J, Gentile C. Prevalência de Anofelinos (Diptera: Culicidae) no crepúsculo vespertino em áreas da Usina Hidrelétrica de Itaipu, no Município de Guaíra, Estado do Paraná, Brasil. Mem Inst Oswaldo Cruz. 1997;92:745-54.

8. Lopes J, Lozovei AL. Ecologia de mosquitos (Diptera: Culicidae) em criadouros naturais e artificiais de área rural do norte do Estado do Paraná, Brasil. I - Coletas ao longo do leito de ribeirão. Rev Saúde Pública. 1995;29:183-91. predominância na composição específica dos mosquitos. Dentre essas, destacam-se algumas de importância em saúde pública, o que denota um constante monitoramento na área ao entorno da represa.

\section{AGRADECIMENTO}

À Companhia Energética de São Paulo (CESP) pela estrutura para o trabalho de campo.

9. Müller AC. Hidrelétricas, meio ambiente e desenvolvimento. São Paulo: Makron Books do Brasil; 1995.

10. Nasci RS. A lightweight battery-powered aspirator for collecting resting mosquitoes in the field. Mosq News. 1981;41:808-11.

11. Natal D, Barata EAMF, Urbinatti P, Barata JMS. Contribuição ao conhecimento da fauna de imaturos de mosquitos (Diptera, Culicidae) em área de implantação de hidrelétrica na bacia do Rio Paraná, Brasil. Rev Bras Entomol. 1995;39:897-9.

12. Natal D, Barata EAMF, Urbinatti P, Barata JMS, Paula MB. Sobre a fauna de mosquitos adultos (Diptera, Culicidae) em área de implantação de hidrelétrica na bacia do Rio Paraná, Brasil. Rev Bras Entomol. 1998;41:213-6.

13. Reinert JF. Mosquito generic and subgeneric abbreviations (Diptera: Culicidae). Mosq Syst. 1975;7:105-10.

14. Service MW. Mosquito ecology: field sampling methods. $2^{\text {nd }}$ ed. London: Applied Scince Publications; 1993.

15. Shannon R. Methods for collecting and feeding mosquitoes in jungle yellow fever studies. Am J Trop Med Hyg. 1939;19:131-40.

16. Wanderley DMV, Andrade JCR, Meneguetti LC, Chinelatto MJ, Dutra AP. Malária no Estado de São Paulo, Brasil, 1980 a 1983. Rev Saúde Pública. 1985;19:28-36.

Artigo baseado na dissertação de mestrado de MB Paula, apresentada à Faculdade de Saúde Pública da Universidade de São Paulo, em 2001. Financiado pela Fundação de Amparo à Pesquisa do Estado de São Paulo (FAPESP, processos n. 96/10014-1 e n. 99/11377-9). 